\title{
A Inserção de Descritores de Tratamento da Informação na Classificação de Recursos Educativos Digitais de um Repositório
}

\author{
Clésia Jordânia Nunes da Costa ${ }^{1}$, Elvis Medeiros de Melo $^{2}$, Dennys Leite Maia ${ }^{2}$ \\ ${ }^{1}$ Departamento de Matemática - Universidade Federal do Rio Grande do Norte (UFRN) \\ Natal - RN - Brasil \\ ${ }^{2}$ Instituto Metrópole Digital - UFRN - Natal - RN - Brasil \\ clesia_j@hotmail.com, elvismedeiros.mm@gmail.com, dennys@imd.ufrn.br
}

\begin{abstract}
The descriptor is a detail of a cognitive skill that is related to a study content in a particular educational stage. The Information Processing this is a recurring theme of mathematics in the lives of students who may be working in an interdisciplinary way. In this way, the present research had a fundamental role in the classification of Learning Objects in the descriptors of Prova Brasil. Of the 330 resources classified, 38 were classified for Information Processing, objects of investigation of this study. They were made available in a repository, offering the teacher a rich feature. With the analysis of the results, we highlight the few resources developed for this topic and few descriptors for the activities of the Information Processing.
\end{abstract}

Resumo. O descritor é uma minúcia de uma habilidade cognitiva que está relacionado a um conteúdo estudado em determinada etapa de ensino. $O$ Tratamento da Informação (TI) trata-se de um conteúdo da matemática recorrente na vida dos estudantes que pode ser trabalhado de forma interdisciplinar. Alinhado à essas ideias, este artigo tem o objetivo de analisar Objetos de Aprendizagem disponibilizados em um repositório para o bloco de conteúdo do TI, usando como base descritores da Prova Brasil. Dos 330 recursos classificados, 38 foram considerados para TI. Eles foram disponibilizados em um repositório, o OBAMA, oferecendo ao professor um recurso mais rico de características. Com a análise dos resultados, destacamos a pouca quantidade recursos desenvolvidos e poucos descritores para atividades específicas do TI.

\section{Introdução}

Diante da informatização que passam várias setores da sociedade, a Educação não pode se ater a essa realidade e precisa explorar as possibilidades para tornar as Tecnologias Digitais de Informação e Comunicação (TDIC) aliadas em processos de ensino e aprendizagem das diferentes áreas do conhecimento. Este artigo é uma continuação de uma pesquisa que catalogou e classificou Objetos de Aprendizagem (OA) para Matemática para um repositório. Os Recursos Educativos Digitais (RED) encontrados foram armazenados no repositório que está em fase de testes, mas já possui algumas funções à disposição de professores, como sistema de busca por OA.

A necessidade de uma educação Matemática focada no aluno e sua realidade se torna essencial à medida que se observa a quantidade de recursos produzidos e disponíveis na atualidade. De acordo com Santos (1996), o uso de TDIC pode incrementar as relações entre educadores e crianças, políticas e educação, contribuindo para aquisição de conhecimento como relevante fator da melhoria social, proporcionando expressões multiculturais e integração universal dos indivíduos. Alguns RED, como os OA, podem contribuir nesse 
sentido na medida em que oportunizam atividades interativas que exploram conteúdos matemáticos.

A integração das TDIC na sala de aula não minimiza a atuação do professor, muito pelo contrário. Quando o professor explora atividades usando OA com seus alunos, ele passa a ter relevância maior como mediador da aprendizagem. Ao docente cabe ser o elo entre o conhecimento apresentado pelo RED e o aluno para garantir que os objetivos de aprendizagem sejam atingidos. O professor possui saberes fundamentais para que práticas com TDIC sejam, efetivamente, educativas. Como afirma Gouvêa (1999, p.4):

\begin{abstract}
O professor será mais importante do que nunca, pois ele precisa se apropriar dessa tecnologia e introduzi-la na sala de aula, no seu dia a dia, da mesma forma que um professor introduziu, um dia, o primeiro livro numa escola e teve de começar a lidar de modo diferente com o conhecimento, sem deixar as outras tecnologias de comunicação de lado. Continuaremos a ensinar e a aprender pela palavra, pelo gesto, pela emoção, pela afetividade, pelos textos lidos e escritos, pela televisão, mas agora também pelo computador, pela informação em tempo real, pela tela em camadas, em janelas que vão se aprofundando às nossas vistas.
\end{abstract}

A demanda para que professores repensem suas práticas de ensino e explorem outros recursos educativos, como os OA, é evidenciada, dentre outros aspectos, pelos baixos índices de proficiência dos estudantes brasileiros. Os resultados apresentados por pesquisas realizadas pelo Sistema de Avaliação a Educação Básica (Saeb) e a Prova Brasil, mostram um cenário bastante preocupante. De acordo com os dados, apenas $39 \%$ dos alunos do 50 ano do Ensino Fundamental e 14\% dos estudantes do $9^{\circ}$ ano do Ensino Fundamental apresentaram uma aprendizagem adequada para Matemática, de acordo com a prova aplicada no ano de 2015.

A referida avaliação é pensada com base nos conteúdos indicados nos Parâmetros Curriculares Nacionais (PCN), que, para Matemática, são organizados em quatro blocos, quais sejam: (i) Espaço e Forma; (ii) Grandezas e Medidas; (iii) Números e Operações/Álgebra e funções e (iv) Tratamento da Informação. Na matriz de referência da Prova Brasil (2011), esses quatro blocos são desmembrados em descritores, que indicam habilidades esperadas para que estudantes tenham desenvolvido ao final de cada ciclo da Educação Básica. Assim, o documento apresenta 28 descritores para o $5^{\circ}$ ano do Ensino Fundamental, 37 para o 9o ano do Ensino Fundamental e 35 para o $3^{\circ}$ ano do Ensino Médio. Cada descritor representa uma habilidade matemática ligada ao conteúdo estudado em sala de aula, proposto pelo bloco a ser explorado pelo professor, servindo como referência para que ele planeje e execute suas aulas com vistas ao desenvolvimento discente.

Nesse sentido, os OA podem contribuir para diversificar as práticas dos professores ao apresentarem atividades que desenvolvam habilidades matemáticas a partir da formulação e teste de ideias. Castro-Filho et al (2016, p. 14) destacam que "[...] um conceito matemático pode influenciar o desenvolvimento de ferramentas tecnológicas, mas posteriormente pode ser influenciado por essa mesma tecnologia”. Segundo a definição de Wiley (2000), um OA pode ser um conteúdo ou recurso digital para aprendizagem de conteúdo específico, cuja característica principal é a reusabilidade. Com base nisso, é possível propor uma articulação entre as TDIC e o processo de aprendizagem de conceitos matemáticos.

A partir dessas ideias, foi desenvolvido o repositório Objetos de Aprendizagem para Matemática $^{1}$ (OBAMA) que catalogou 514 OA do tipo animação e simulação, que exploram

\footnotetext{
${ }^{1}$ https://obama.imd.ufrn.br/. Último acesso em: 12/06/2017.
} 
conceitos matemáticos da Educação Básica, e classificou em nível de ensino, bloco de conteúdo e pelos descritores que são propostos pela Prova Brasil (2011). Focaremos neste artigo os descritores voltados para o bloco de conteúdo Tratamento da Informação (TI), que se apresentou como um dos blocos mais carentes de OA para todos os níveis de ensino [Oliveira et al 2017], caracterizando deficiência de RED voltados a essa área.

Diante do exposto, objetivo desse trabalho é analisar os OA disponibilizados no OBAMA para o bloco de conteúdo TI, usando como base os descritores usados para a classificação. Com isso, esperamos dispor a professores que ensinam Matemática maior qualidade à avaliação dos OA com menor quantidade para que atenda, cada vez mais, as habilidades específicas para aquele tema tratado em sala.

Este artigo se organiza em cinco seções: Além desta Introdução; a Fundamentação teórica, onde trazemos discussões a respeito do uso dos descritores na classificação dos OA, em específico ligadas a este estudo; Metodologia, em que mostramos os passos do trabalho realizado; Resultados e discussões, onde analisamos os dados obtidos a partir da classificação dos OA; e finalizamos com as Conclusões que, a partir das análises e estudos, foram permitidas realizar.

\section{Fundamentação teórica}

D’Ambrósio (1991) afirma que há algo errado com a Matemática que estamos ensinando. O conteúdo que tentamos passar adiante por meio dos sistemas escolares é obsoleto, desinteressante e inútil. De acordo com o referido pesquisador, o ensino de Matemática precisa de uma mudança, deixar o tradicionalismo e introduzir novas metodologias, pois ela é vista como uma disciplina que desperta medo e preocupação nos alunos. Uma dessas metodologias é a inserção das TDIC nas aulas de Matemática, vista como uma tendência no ensino da disciplina em razão da ampliação de possibilidades didáticas que elas proporcionam.

O desenvolvimento da informatização na sociedade vem associado à utilização da informática no ambiente escolar. Há quase duas décadas, Valente (1998) já alertava que o termo Informática na Educação refere-se à inserção do computador no processo de aprendizagem dos conteúdos curriculares de todos os níveis e modalidades de educação. Nesse sentido, o uso do computador nas salas de aula deve ser visto como estratégia que auxilie o docente a proporcionar um aprendizado crítico, reflexivo e criativo, que desperte a atenção e curiosidade dos alunos. Conhecer e avaliar os RED, especialmente no que tange aos aspectos pedagógicos, é fundamental para a efetivação dessa proposta pelos professores da Educação Básica. A possibilidade de explorar um conteúdo matemático, por meio do uso de OA, pode transcender as barreiras que a educação vem tentando superar.

As diretrizes para a Educação Matemática [Brasil 1997] defendem um ensino por meio do desenvolvimento integrado dos conhecimentos. Assim, constata-se a necessidade de um trabalho mais efetivo com os gráficos, relacionando o $\mathrm{TI}$, o conhecimento diário e a Matemática, além de outros conhecimentos curriculares como Ciências, Língua Portuguesa, Geografia, História, dentre outros. O TI é um conhecimento social explorado por todos os tipos de mídia e da interdisciplinaridade trazendo ao estudante uma ligação entre o componentes curriculares apresentados e a Matemática como ilustradora dos dados apresentados. De acordo com Pagan e Magina (2011), a interdisciplinaridade contribui para a aprendizagem de conceitos elementares da estatística, pois trabalhar os conceitos que envolvem a construção e a compreensão de gráficos de forma contextualizada favorece o desenvolvimento de competência de tal modo que situa os estudantes em um campo mais amplo do conhecimento, possibilitando maior compreensão das informações ao seu redor. 
Pesquisas como as de Ainley, Nardi e Pratt (2000); Magina e Santos (2008) e Castro et al (2011) revelam que o estudo de gráficos com auxílio do ambiente computacional contribui para a aprendizagem de conceitos estatísticos. Conforme essas pesquisas, as TDIC, na maioria das vezes, auxiliam na visualização e exploração de um conjunto de atividades, propiciando novas formas de pensar e agir. Estes aspectos motivaram a produção do RED que explora os conceitos de TI, apresentado por Silva et al (2015).

O trabalho de Castro e Castro-Filho (2012) explorou habilidades de TI e mostrou um ambiente promissor para o desenvolvimento de atividades interdisciplinares e produção de conteúdo, viabilizando a liberdade produtiva das crianças, que desenvolveram a capacidade argumentativa. De acordo com os dados da pesquisa, a experiência de uso de TDIC para a aprendizagem de TI ampliou os procedimentos e estratégias de coleta, organização de dados e comunicação, além de desenvolver o senso crítico, a autonomia, a criatividade e a leitura das crianças o que favoreceu a análise e a interpretação de textos, imagens e gráficos. Isso foi oportunizado pela mobilidade proporcionada pelos laptops, utilizados no modelo 1:1 (um dispositivo por aluno) que permitiu o uso de OA e um blog para a realização das atividades.

O descritor é uma associação entre conteúdos curriculares e operações mentais desenvolvidas pelo aluno, que traduzem certas competências e habilidades. Os descritores indicam habilidades gerais que se esperam dos alunos e constituem a referência para seleção dos itens que devem compor uma prova de avaliação. Analisando o conteúdo desses descritores, vemos que eles expressam atividades pontuais, em que o professor deve desenvolver em salas de aula como seus objetivos para determinados níveis de ensino. Estas habilidades matemáticas são sugeridas nos documentos oficiais no Saeb do Plano de Desenvolvimento da Educação (PDE), como referência para a elaboração de itens avaliativos da Prova Brasil. O quadro 01, a seguir, mostra os descritores por níveis de ensino.

\section{Quadro 01. Descritores por nível de ensino}

\begin{tabular}{|c|c|}
\hline \multicolumn{2}{|r|}{ Anos Iniciais do Ensino Fundamental (5a ano) } \\
\hline D27 & Ler informações e dados apresentados em tabelas \\
\hline D28 & Ler informações e dados apresentados em gráficos (particularmente em gráficos de colunas) \\
\hline \multicolumn{2}{|r|}{ Anos Finais do Ensino Fundamental (9o ano) } \\
\hline D36 & Resolver problemas envolvendo informações apresentadas em tabelas e/ou gráficos \\
\hline D37 & $\begin{array}{l}\text { Associar informações apresentadas em listas e/ou tabelas simples aos gráficos quais as representam } \\
\text { e vice-versa }\end{array}$ \\
\hline \multicolumn{2}{|r|}{ Ensino Médio ( $3^{\circ}$ ano) } \\
\hline D34 & Resolver problemas envolvendo informações apresentadas em tabelas e/ou gráficos \\
\hline D35 & $\begin{array}{l}\text { Associar informações apresentadas em listas e/ou tabelas simples aos gráficos quais as representam } \\
\text { e vice-versa }\end{array}$ \\
\hline
\end{tabular}

Para o ensino e a aprendizagem da Matemática, Castro-Filho et al (2016) destacam que o acesso a diferentes fontes de informação, as múltiplas formas de representar o pensamento matemático, a manipulação dinâmica de símbolos matemáticos são alguns dos elementos que favorecem o pensamento matemático apoiado por TDIC. O professor deve estar preparado para conhecer especificidades das TDIC para incluí-las em seu plano de aula e oportunizar práticas, efetivamente inovadoras com seus alunos. Ao incluir atividades com 
base nos descritores, o docente norteará sua prática e desenvolver um trabalho mais voltado para demandas cognitivas de seus alunos, tendo em vista que os descritores tratam de uma habilidade ligada a um tema curricular. É mais uma possibilidade de enriquecimento da aula do professor ao se olhar para os OA como ferramentas de potencial em sua sequência didática.

Nesse sentido o repositório OBAMA atende às demandas do professor quando permite a busca por OA a partir de objetivos pensando para sua aula. Ao classificar os OA por descritores, o OBAMA ajuda o professor na elaboração de seu planejamento de aulas, direcionando a escolha de recursos indicados para as habilidades que ele deseja desenvolver com seu aluno. Na seção seguinte, apresentamos a metodologia que orientou a execução e análises deste trabalho.

\section{Metodologia}

Essa pesquisa possui abordagem mista, com foco em dados quantitativos e qualitativos, tendo em vista a natureza dos dados em análise. Foram usados cálculos estatísticos descritivos e construção de tabelas para possibilitar o desenvolvimento de gráficos como produto visual. Portanto, separamos quantitativamente os OA para TI listados no OBAMA, em categorias como níveis de ensino e descritores. $\mathrm{O}$ aspecto qualitativo ocorreu a partir da classificação e avaliação realizadas pelos pesquisadores, ao acessarem os OA.

Considerando que a plataforma do repositório ainda possui algumas funções em implementação, para registro e análise neste artigo, selecionamos os OA para desktop encontrados, catalogados e classificados até fevereiro de 2017. Atualmente, os recursos são apresentados por meio de uma busca, em que o usuário pode escolher filtrar por níveis de ensino, bloco de conteúdos e descritores, que são o objeto de estudo deste artigo. No repositório há recursos para desktop e mobile. Escolhemos fazer as análises para recursos desktop, pois, além de compor a maioria dos OA disponíveis, a busca detalhada para os recursos mobile ainda está em desenvolvimento.

Do total de OA catalogados e classificados para o OBAMA, presentes em Oliveira et al (2017), 330 recursos para desktop foram classificados de acordo com os descritores. Vale salientar que só existem descritores para a série final de cada nível de ensino, e que estes foram utilizados como referência para classificá-los. Além disso, não há descritores para a Educação Infantil nos documentos oficiais do Plano de Desenvolvimento da Educação (PDE).

Com base nos dados da primeira classificação, nos atentamos ao bloco de conteúdo TI que se apresentou como bloco com menor número de OA disponível. A classificação com base nos descritores da Prova Brasil (2011) foi uma forma de visualizar as possibilidades de uso das TDIC como ferramentas de ensino e aprendizagem, com um maior detalhamento da potencialidade do OA.

Dos 514 recursos catalogados e classificados para o OBAMA, destacamos que apenas 61 OA foram classificados dentro do tema TI. Dos 61 recursos dentro tema, 38 OA são para desktop e serão considerados o universo dessa análise. Alguns OA foram reclassificados na fase de adição dos descritores, dando continuidade ao trabalho de Oliveira et al (2017), por se adequar melhor para alguns objetivos do que a outros.

Em posse dos OA já catalogados e classificados por blocos de conteúdo, dentro do repositório OBAMA, e por níveis de ensino - anos iniciais e finais do Ensino Fundamental e Ensino Médio -, seguimos para a classificação com base nos descritores. A classificação dos OA em cada um dos descritores disponíveis foi determinada de acordo com o bloco a qual eles estavam inseridos e as características apresentadas ao serem testados. No entanto, houve OA que pertencia a um bloco de conteúdo, mas não era contemplado por nenhum descritor. Para contabilizar esses recursos criamos uma nova categoria, a DO - Não Identificado, 
reunindo os OA que, diante da análise e observação dos descritores, não se enquadraram em nenhum deles. A seguir, apresentamos os resultados e discussões da pesquisa.

\section{Resultados e Discussões}

O repositório OBAMA tem como proposta oferecer a professores que ensinam Matemática na Educação Básica um ambiente que oportunize acesso, em um único endereço web, ao maior número de OA para as suas aulas, com a indicação da etapa de ensino, tema curricular e a confiabilidade de características educativas do recurso. No levantamento atual, foram incluídos, nos OA para desktop já presentes no repositório, descritores com a finalidade de enriquecer a busca por um determinado recurso. Apresentamos como os descritores de TI são apresentados nos OA catalogados no OBAMA e distribuídos por níveis de ensino.

Iniciamos com as análises de OA para TI por níveis de ensino da Educação Básica. O Gráfico 1 mostra a quantidade final de OA no bloco de conteúdo TI, distribuídos por nível de ensino, levando em consideração que um OA pode estar em mais de um nível.

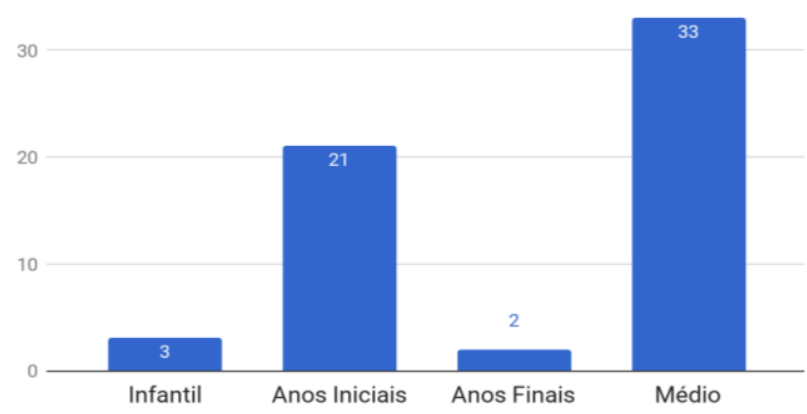

Gráfico 1. Quantidade Final de OA no Bloco de conteúdo TI por nível de ensino.

É possível observar que dos OA classificados dentro do bloco de conteúdos do TI, existem mais recursos desenvolvidos para os anos iniciais do Ensino Fundamental e para o Ensino Médio, comparando com as outras áreas. A baixa quantidade de OA encontrados para os anos finais do Ensino Fundamental reforça a necessidade do desenvolvimento de OA, para o campo do TI, tendo em vista as potencialidades dessas ferramentas. Na fase de análise dos descritores, não levamos em consideração a Educação Infantil, por não haver descritor, apesar de encontrarmos três recursos que se adequaram a esse nível. Isto, aliás, é um dos aspectos que destacamos no OBAMA pois desvela e amplia as possibilidades de reutilização do OA, considerando, inclusive, outros níveis.

Prosseguindo a análise, classificamos os 38 OA também quanto aos respectivos descritores de cada um dos níveis de ensino, segundo Prova Brasil (2011). Primeiramente, analisamos o Gráfico 2 que mostra os OA classificados dentro dos descritores para os anos iniciais do Ensino Fundamental, levando em consideração que um OA pode estar em mais de um descritor.

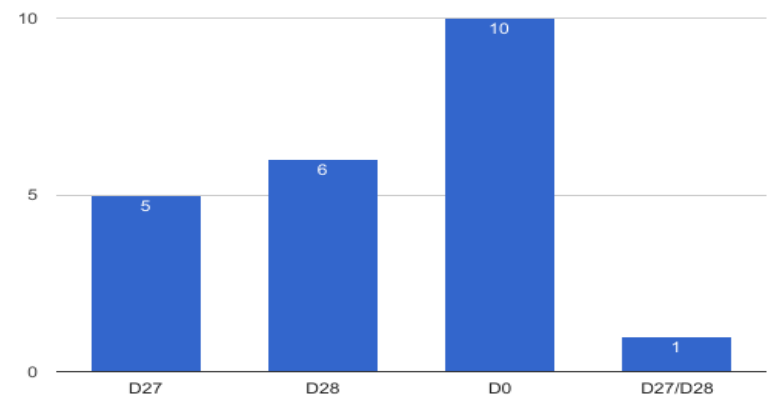

Gráfico 2. Quantidade de OA por descritor dos anos iniciais do Ensino Fundamental. 
De acordo com o gráfico, há um equilíbrio entre o número de OA aptos para os dois descritores do TI. Contudo, vale registrar a quantidade elevada, correspondente ao equivalente para os dois descritores juntos, de OA sem nenhum descritor. Cerca de $50 \%$ dos OA classificados dentro do bloco de conteúdos do TI não se enquadraram em nenhum descritor. Este dado indica uma revisão da classificação ou mesmo que as habilidades desenvolvidas pelo OA não sejam mensuradas pelo sistema de avaliação da Prova Brasil. Afinal, o modelo de prova restringe as possibilidades de avaliação de algumas competências, como é o caso, por exemplo, de estimativas que exploram cálculo mental.

Como exemplo de OA para esse nível de ensino, tem-se o recurso Viajando com a Matemática (Figura 1). Esse RED foi classificado dentro do bloco de conteúdos do TI, com o descritor D28 - Ler informações e dados apresentados em gráficos (particularmente em gráficos de colunas). Trata-se de um OA no qual o usuário precisa fazer uma viagem por todas as cidades. Para isso, ele precisa analisar informações em gráficos e fazer os cálculos necessários para concluir as atividades. A Figura 1 ilustra a interface do OA.

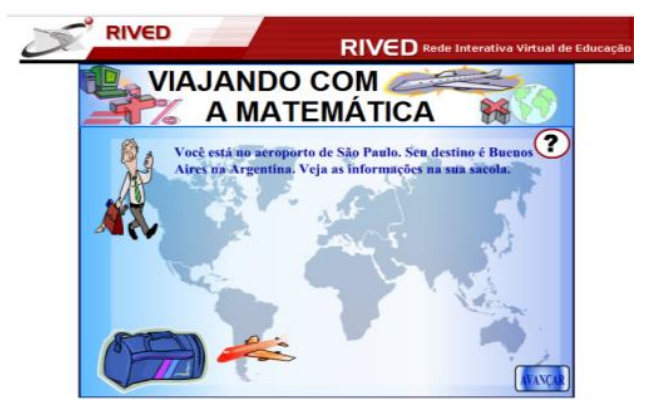

Figura 1. Tela do OA Viajando com a Matemática.

A seguir (Gráfico 3) a classificação dos OA para os anos finais do Ensino Fundamental, portanto, para alunos do $6^{-}$ao $9^{\circ}$ ano, levando em consideração que um OA pode estar em mais de um descritor.

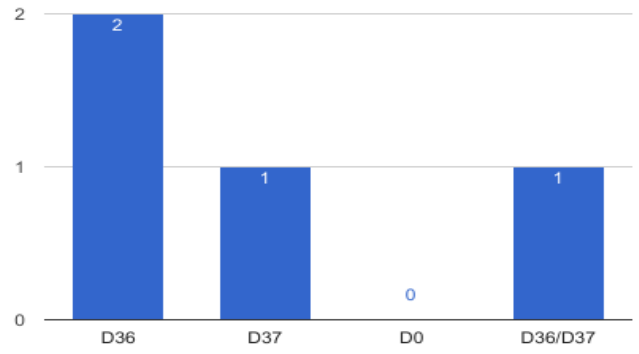

\section{Gráfico 3. Quantidade de OA por descritor dos anos finais do Ensino Fundamental.}

Nesse nível de ensino, há um déficit quanto ao número de recursos desenvolvidos para o bloco de conteúdos do TI. Encontramos apenas dois OA, sendo que os dois se enquadraram no D36 e um se encaixou no descritor D37. Dentre os dois, destacamos o OA que estava em todos os descritores para esse nível de ensino. O OA Água em Jogo, da FabriCO. A Figura 2 ilustra a sua interface. 


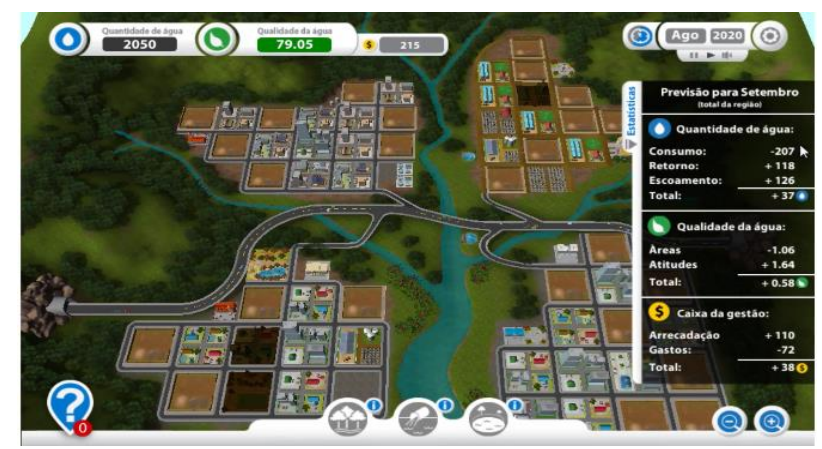

Figura 2. Tela do OA “Água em Jogo", da Fabrico.

Esse OA tem o objetivo de simular os impactos das ações do homem sobre os recursos hídricos de uma bacia hidrográfica. O desafio lançado é garantir que água em quantidade e qualidade sejam distribuídas de maneira adequada, a fim de atender as necessidades das áreas residenciais, industriais, comerciais e rurais, além disso, garantir o abastecimento para as futuras gerações. Ele traz atividades de gerenciamento e, em sua interface, mostra-se tabelas e gráficos em que o usuário precisa interpretá-las para chegar ao objetivo do OA.

Por fim, o Gráfico 4 ilustra a quantidade de OA do Ensino Médio e seus respectivos descritores, levando em consideração que um OA pode estar em mais de um descritor.

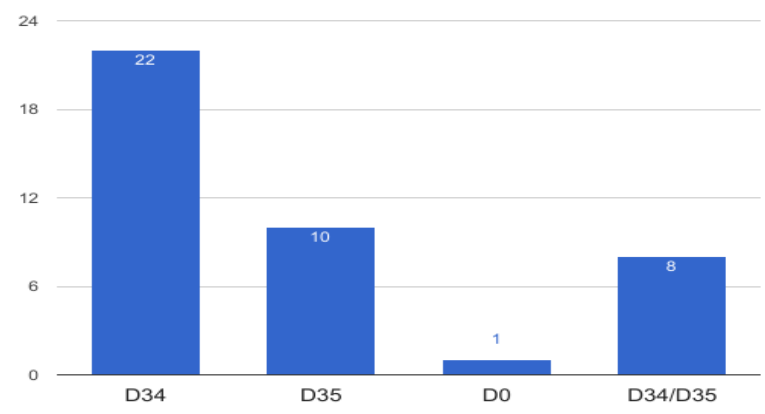

Gráfico 3. Quantidade de OA por descritor do Ensino Médio.

Para esse nível de ensino, comparado aos demais, existem mais OA do que para qualquer outro nível. Este dado sugere que o Ensino Médio é a etapa da Educação Básica que tem maior visibilidade por desenvolvedores. Encontramos mais OA que contemplam o descritor D34 se comparado ao descritor D35. Desse fato podemos inferir que existem poucos recursos que explorem a competência de interpretação ou construção de gráficos a partir de tabelas, tão importante para o cotidiano dos alunos.

Um exemplo de recurso do descritor D34 é o OA Combinações. Na Figura 3, tem-se uma atividade envolvendo ciclistas e formação de duplas.

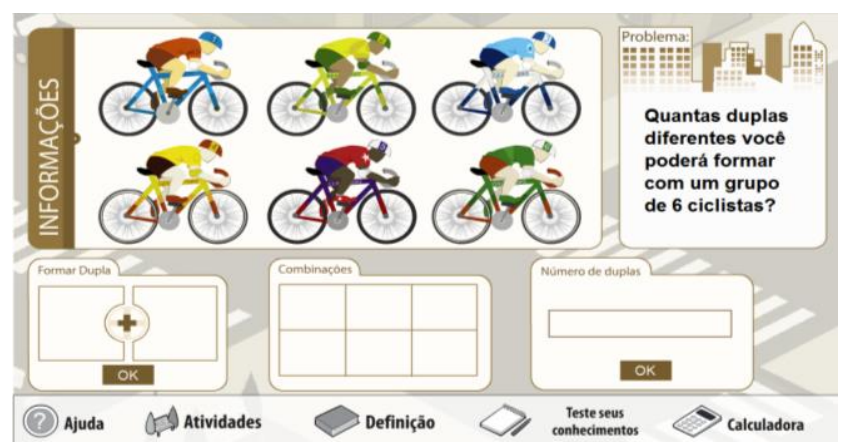

Figura 3. Tela do OA Combinações. 
Trata-se de um OA com atividades envolvendo combinatória, em que o usuário precisa resolver as situações-problema colocadas para ir avançando de nível. Para isso, em sua interface possui recursos como calculadora, além de tabelas que esquematizam as respostas do usuário, assim como escolha dos problemas que ele deseja resolver. $\mathrm{O}$ usuário precisa interagir com o recurso das tabelas para poder avançar na resolução dos problemas do OA.

A existência de OA com potencialidades para dois descritores evidencia a correlação de conceitos e áreas dentro da Matemática. Os recursos, classificados para esse nível de ensino, trazem atividades mais específicas dentro de descritores. Por terem atividades que exigem um conhecimento interdisciplinar, é intrínseca aos seus conteúdos abordagens que precisem da interpretação tanto de tabelas como de gráficos. Isso justifica o fato de que, aproximadamente, $30 \%$ dos recursos possuem todos os descritores deste nível de ensino.

Como exemplo, o OA Aviões e Matrizes aborda como as matrizes podem ser utilizadas na análise e na elaboração de malhas aéreas. Essa aplicação consiste num exemplo prático do produto de matrizes, introduzindo também o conceito de grafo. As atividades do recurso trazem, na sua interface, tabelas, gráficos, assim como esquemas em que o usuário precisa interpretar as informações para poder ir prosseguindo com as atividades. A Figura 4 traz a imagem de uma questão presente no OA.
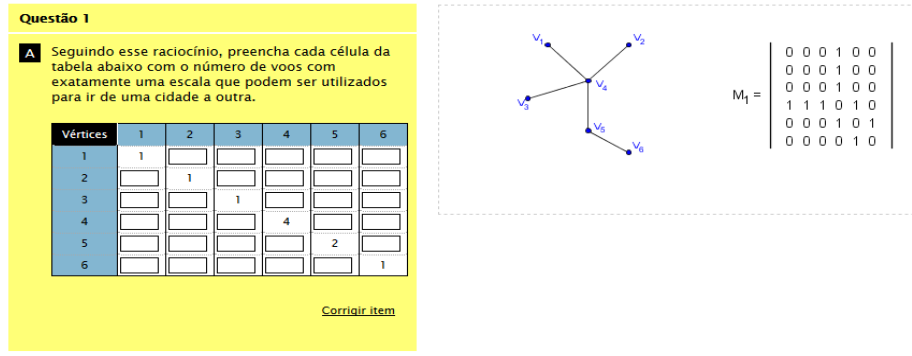

Figura 4. OA Aviões e Matrizes.

A seguir, apresentamos as conclusões deste trabalho, a partir das inferências que fizemos sobre os dados analisados.

\section{Conclusões}

De maneira geral a nossa pesquisa traz um campo de estudo e inovação para o ensino de Matemática que precisa ser investigado e explorado. A popularização da informação de forma instantânea e dos RED, como os OA, demandam aos professores da atualidade conhecer melhor seus alunos e as possibilidades pedagógicas que podem se servir. A catalogação desses recursos e classificação em descritores contribui para a prática docente na medida em que facilitará seu trabalho ao planejar e executar uma aula com suporte de TDIC.

Por meio dos dados encontrados e das discussões feitas a partir deles, podemos concluir que há poucos recursos desenvolvidos para TI, apesar de este bloco ter apenas dois descritores para cada nível, com objetivos generalistas. As atividades encontradas nos OA para esse tema são muito complexas ou específicas. Se faz necessário desenvolvimento de novos recursos, além de revisão dos objetivos para essa área em matrizes nacionais que avaliam a Educação Básica. Isso poderá potencializar os processos de ensino e aprendizagem da Matemática.

Seguiremos o projeto com uma formação docente e disponibilizar planos de aula de professores, tornando o repositório uma ferramenta de contínua colaboração. Em etapas seguintes da pesquisa, pretendemos observar e promover reflexões mais profundas sobre os impactos provocados pelo uso dos OA no processo de ensino e aprendizagem da Matemática 
VI Congresso Brasileiro de Informática na Educação (CBIE 2017)

Anais do XXIII Workshop de Informática na Escola (WIE 2017)

na Educação Básica, assim como elaboração de OA específicos para a área do Tratamento da Informação.

\section{Referências}

Ainley, J., Nardi, E.; Pratt, D. (2000). "Towards the construction of meaning for trend in Active Graphing, International Journal of Computers for Mathematical Learning"; 5.2, p. 85-114.

Brasil (2011). "Prova Brasil: Ensino fundamental - matrizes de referência, tópicos e descritores." In: Brasil. PDE: Plano de Desenvolvimento da Educação. Brasília: MEC/SEB/Inep.

Brasil (1997). Parâmetros curriculares nacionais: Matemática. Brasília: MEC/Secretaria de Educação Fundamental.

Castro, J.; Castro-Filho, J. A. (2012). Projeto Um Mundo de Informações: integração de tecnologias digitais ao currículo escolar. In: Anais dos Workshops do CBIE 2012 Workshop sobre formação e experiências educacionais no programa Um Computador por Aluno. Porto Alegre: Sociedade Brasileira de Computação, v. 1. p. 1-10.

Castro-Filho, J. A.; Maia, D. L.; Castro, J. B. de; Barreto, A. L. de O.; Freire, R. S. (2016). "Das tabuletas aos tablets: tecnologias e aprendizagem da Matemática". In: Castro-Filho, J. A. et al. (Orgs.). Matemática, Cultura e Tecnologia: perspectivas internacionais. Curitiba: CRV, p.13-34.

Castro et al (2011). Objetos de Aprendizagem digitais como suporte para a construção e compreensão de gráficos. In: Anais da XIII CIEAEM. Recife: Edumatec/UFPE.

D’Ambrósio, U. (1991) “As matemáticas e seu entorno sócio-cultural”. In: Memorias del Primer Congreso Iberoamericano de Educación Matemática, Paris.

Gouvêa, S. F. (1999) "Os caminhos do professor na era da tecnologia”. Revista de educação e informática, a. 9, n. 13, abril.

Oliveira, A. M. D; Silva, A. C. N.; Costa, C. J. N.; Maia, D. L. Levantamento e catalogação de objetos de aprendizagem para Matemática para atualização de um repositório. In: Anais do Ctrl+E 2017, Mamanguape, PB: UFPB.

Pagan, M. A.; Magina, S. M. P. (2011). A Interdisciplinaridade auxiliando o ensino da estatística na Educação Básica. In: Anais da XIII CIEAEM. Recife: Edumatec/UFPE.

Santos, N. (1996) A natureza do espaço, técnica e tempo, razão e emoção. São Paulo:Hucitec.

Santos, S. S; Magina, S. M. P. Estratégias de Interpretação Gráfica de uma Professora Polivalente ao Manipular Dados no Ambiente Computacional. Revista Bolema, Rio Claro (SP), Ano 21, nº 29, 2008, pp. 157 a 174.

Silva et al (2016). Esquadrão Graphics: em repórter por um dia. In: Anais dos Workshops do CBIE 2015. Maceió: UFAL.

Valente, J. A. (1998) "Diferentes usos do computador na educação". In: Diferentes usos do computador na educação. O uso inteligente do computador na educação.

Wiley, D. A. (2000). "Connecting learning objects to instructional design theory: a definition, a metaphor, and a taxonomy”. Logan: Utah State University. 